\title{
Hypoxic tubular epithelial cells regulating angiogenesis by Rab 7
}

Yiqiong Yang ${ }^{1}$, Jing Wang ${ }^{2}$, Yu Zhang ${ }^{1}$, Xiuxiu $\mathrm{Hu}^{1}$, $\mathrm{Li} \mathrm{Li}^{1}$, Pingsheng Chen ${ }^{3, *}$

1. Department of Pathology and Pathophysiology, Medical School, Southeast University, Dingjiaqiao 87, Gulou District, Nanjing 210009, China

2. Institute of Andrology, The Affiliated Drum Tower Hospital, Nanjing University, Nanjing, People's Republic of China

3. Institute of Nephrology, The Affiliated Zhongda Hospital, Southeast University, Nanjing, People's Republic of China

*: Corresponding Author

This study was supported by the National Natural Scientific Foundation of China (No. 81370868) and Key R \& D Program of Jiangsu Province, China (No BE2019712).

\begin{abstract}
Aim: The purpose of our study was to discuss Rab 7 effects in chronic kidney disease (CKD).

Methods: Using WT and Rab 7-/- mice as target animal, and HK-2 and HMEC-1 cell co-cultured to make cell model. Measuring kidney tissues were evaluated by Sirius red staining, immunohistochemistry staining to CD 34 protein, Transmission electron microscope (TEM) and gelatin zymography to MMP-2 activities. The cell proliferation were measured by CCK-8 and Ki67 protein expression. Measuring cell invasion and total length were evaluated by transwell and in vitro angiogenesis assay. MMP-2 activities were evaluated by gelatin zymography in cell groups. The relative proteins expression were evaluated by Western blot in kidney tissues and cell groups.

Results: Hypoxia promoted the expression of Rab7 in HMEC-1, and the activity of MMP-2 related with regulatory molecules such as reversion-inducing-cysteine-rich protein with kazal motifs (RECK), negative correlation with membrane-type $1 \mathrm{MMP}$ (MT1-MMP or MMP-14) on the membrane of TECs. In addition, the up-regulation of the expression of Rab7 inhibited the activity of MMP-2 and proliferation and cyclization of endothelial cells, and the inhibitor of MMP-2 partially blocked the effects of Rab7 on angiogenesis. Furthermore, the similar data were also obtained in the fibrosis kidney tissues of mice.

Conclusion: Rab 7 might be an important role in hypoxic TECs regulated angiogenesis, Rab 7 knockdown could improve hypoxic TECs regulated angiogenesis, the relative mechanisms might be correlation with RECK pathway and MMP-2 activities in vivo and vitro study.
\end{abstract}

Key words: Rab 7; hypoxic; HMEC-1; MMP-2 activity; RECK 
Running Title: Rab 7 and kidney fibrosis

\section{Introduction}

CKD has already been a serious public health problem with more than 70million reported cases affected [1]. Although the prevalence of CKD varies in different countries and regions but the prevalence of CKD in high-income countries such as United States and Australia has been around 11\%. The etiology of CKD varies such as the deposition of immune complex, hypertension, and hyperglycemia and so on and then it causes kidney cell damage and chronic inflammation with fibroblasts activation and extracellular matrix synthesis increases, eventually results in renal interstitial fibrosis and finally transforms CKD to end-stage renal disease (ESRD). There are still many terrae incognita badly in need of further study on account of complicated renal fibrosis mechanism. [2].

Peritubular capillary reduction is a prominent pathological phenomenon in the progression of CKD, but the mechanism is still unclear. The renal tubular epithelial cells (TECs) are the main cells of kidney and extremely sensitive to hypoxia, proteinuria, toxins, metabolic disorders and senescence. It can release large numbers of active substances which are the primary causes of CKD and may perform different functions in status of peritubular capillaries.[3] It is believed that the decrease of peritubular capillaries may related to main factors as follows: Firstly, decreased blood flow through the peritubular capillaries decreased shear force on vascular wall and then weakened survival signals for blood flow-dependent endothelial and in the end caused endothelial cell apoptosis. Secondly, the pericyte cells detached from the wall of the blood vessel because of local inflammation followed by increased capillary permeability and instability and ultimately caused capillary destruction. Thirdly, the microenvironment of peritubular capillaries changed in advanced disease, especially large amount of type I and type III collagen instead of less extracellular matrix and hindered exchange of information between cells and obstructed the status of VEGF[46]. It can be seen from above that there are many reasons for decrease of peritubular capillaries and the effects of renal tubular epithelial cells injury on the formation of peritubular capillary remained to be further studied.

Previous studies showed that VEGF and MMP-2 were key factors of angiogenesis [7]. If hypoxic renal tubular epithelial cells were associated with these two molecules, angiogenesis might be affected. Our previous study found that renal tubular epithelial cells in hypoxic condition could express excessive production of VEGF and MMP-2, but MMP-2 activity was decreased [2,8]. Therefore, the main reason for the decrease of renal fibrosis tissue capillaries might be related to the decrease of the activity of MMP-2. So we started to focus on the regulation of MMP-2 activity.

MMP-2 is a key role in MMPs. A variety of molecules can regulate MMP-2 activity, such as TIMP-2, RECK and MMP-14. TIMP-2 can mediate MMP-14 to bind with pro-MMP-2 to form a complex to promote MMP-2 activity in low concentration; on the other hand, TIMP-2 overexpression could depress MMP-2 activity [9]. RECK protein in cell membrane could down-regulate MMP-2 activity. MMP-2 has a wide range of functions after activation and can be degraded up to 27 extracellular substances. It can also play an anti-inflammatory role by antagonizing phospholipase A2 and monocyte chemotactic protein $3[10]$.

Since the activation of MMP-2 is mainly carried out on the surface of plasma membrane, will the membrane structure change affect the activity of this enzyme? Autophagy and endocytosis can induce cell membrane remodeling and regulate 
transmembrane signal transduction. Hypoxia can enhance autophagy and endocytosis $[2,8]$, especially membrane receptor-related endocytosis [11]. Endocytosis and autophagy co-occur with sharing functional links in hypoxia condition [2].

Rab7 has been proved to be one of the common molecules of endocytosis and autophagy [11]. Some research had found that Rab7 is a key member in endosomal membrane transportation. In addition, Rab7 has been proved to be related to autophagy vesicles and is essential for the maintenance of physiological autophagy [12]. Rab7 could regulate endocytosis and autophagy, and participated in the fusion of autophagy endocytosis and lysosomes. In conclusion, Rab7 has been proved to be a key regulator of endocytosis and autophagy [13]. The intervention of the shared molecules may affect the activity of MMP-2 and thereby affect angiogenesis in CKD.

The abnormal expression of Rab7 in hypoxic renal tubular epithelial cells may cause remodeling of cell membranes and lead to decreased activation of MMP-2 and thereby interfering with angiogenesis. This work intends to confirm whether Rab7 regulates angiogenesis through MMP-2 and its internal mechanism.

\section{Materials and Methods \\ Mice and Reagents}

Our research was approved by the Animal Care Committee of Southeast University (No.20170306003). Rab7 knockout mice were constructed by using CRISPR/Cas9 technology (SaiyeBiotechnology Co, LTD.).Wild-type mice (C57BL/6) (Qinglongshan Experimental Animal Company, Nanjing, China). Mice were raised in a facility with controlled air, temperature and light. At the beginning of the experiment, the mice weighed $18-22 \mathrm{~g}$. The mice were provided with a normal diet (XieTong, Nanjing, China) and tap water throughout the experiment. CsA (Chem Best, Shanghai, China) was diluted to $10 \mathrm{mg} / \mathrm{mL}$ in sunflower oil (Arawana, Shanghai, China). Furosemide (ZhaoHui, Shanghai, China) was diluted to $10 \mathrm{mg} / \mathrm{mL}$ in distilled water.

\section{Experimental Design}

Dividing mice as 4 groups of 5 mice as follows: WT, WT model and Rab7-/- model. In order to induce chronic renal fibrosis, mice in model groups received the following interventions: $50 \mathrm{mg} / \mathrm{kg}$ furosemide 2 days before the experiment, followed by daily administration of $25 \mathrm{mg} / \mathrm{kg}$ CsA and $50 \mathrm{mg} / \mathrm{kg}$ furosemide on alternate days for 28 days. Mice in WT Group was untreated for all 28 days. The body weight of each rat was recorded every 4 days and the dosage was adjusted accordingly.

After 28 days, mice were anaesthetized with pentobarbital (Xiya, Linyi, China) and sacrificed. Kidneys were obtained for histology and western blot detection.

\section{Establishing of HK-2-Rab7- cell line (stably lower expression of Rab7)}

HK-2 cells were seeded into 96-well plates with $1.0 \times 10^{6}$ cells per well. Rab7 lentiviral vector was successfully constructed. Then, HK-2 cells were transfected with si-Rab7 or si-NC (negative control). Finally, quantitative real-time PCR and western blot analysis were performed to confirm the expression of Rab7, the data were not shown in study.

\section{Co-culture experiments}

HMEC- 1 cell density was adjusted to $2 \times 10^{5} / \mathrm{mL}$. Cell suspension $(100 \mu \mathrm{L})$ was added to each well of 96-well plate. HMEC-1 cell were co-cultured with HK-2, HK-2 with si-NC or HK-2 with si-Rab 7. Cell groups were divided into NC group (HK-2 and HMEC-1 cell were co-cultured in normoxia), Model group (HK-2 and HMEC-1 cell 
were co-cultured in hypoxia), Model+si-Rab 7 group (HK-2 with si-Rab 7 and HMEC1 cell were co-cultured in hypoxia), Model+si-Rab 7+MMP-2 inhibitor (HK-2 with siRab 7 and HMEC-1 cell were co-cultured and treated with MMP-2 inhibitor in hypoxia). The hypoxia was $94 \% \mathrm{~N}_{2} / 5 \% \mathrm{CO}_{2} / 1 \% \mathrm{O}_{2}$.

\section{CCK-8 Cytotoxicity Experiment}

The cell were treated by difference methods, After $0.5 \mathrm{~h}, 1 \mathrm{~h}, 2 \mathrm{~h}$ and $3.5 \mathrm{~h}, \mathrm{CCK}-8$ dye was added to each well and the plates were incubated for another $2 \mathrm{~h}$ at $37{ }^{\circ} \mathrm{C}$, optical density (OD) values were measured at $450 \mathrm{~nm}$ by a micro plate reader (Biotek, Winooski, VT, USA) and measured the cell proliferation.

\section{Immunofluorescence}

After treatment, fixing cells by $4 \%$ par formaldehyde at $1 \mathrm{~h}$ and permeabilizing by $0.5 \%$ Triton X-100 at 10 min. Using 10\% sheep serum (BOSTER, China) to block nonspecific binding. Incubation by antibodies against Ki67 (1:200) (Proteintech, China) at $4^{\circ} \mathrm{C}$ overnight, adding secondary antibody $(1: 1000)$ (Thermo Fisher Scientific, USA) to apply for $1 \mathrm{~h}$. Using DAPI to stain nuclear for $5 \mathrm{~min}$. Capturing image by laser scanning con focal microscopy (Olympus Corporation, Japan), evaluating Ki67 positive cell number of difference groups.

\section{Transwell assays}

The HMEC-1 cell were treated by difference methods for $24 \mathrm{~h}$, Adding medium $(50 \mu \mathrm{L})$ to upper well of the transwell chamber containing $2.5 \times 10^{3}$ cells/well and above medium with $20 \%$ FBS in the lower site. Incubation for $48 \mathrm{~h}$, cells in the upper chamber were removed and cells in the lower chamber had been stained with crystal violet at room temperature for $30 \mathrm{~min}$. Finally, cells in the lower chamber were counted under a light microscope. Each experiment had been repeated at least three times.

\section{In vitro angiogenesis assay}

Adjusting HMEC-1 cell density to $2 \times 10^{5} / \mathrm{mL}$. Adding Cell suspension $(100 \mu \mathrm{L})$ to 96 -well plate. Culturing cell for $2 \mathrm{~h}$ by difference methods. The capillary-like tubule networks were analyzed by measuring total length of the cells per field by Image $J$ Software. The experiment had been repeated for three times.

\section{Western blot (WB)}

Collecting kidney tissues or culture cells and extracting protein. Measuring total protein by a BCA protein assay kit (Beyotime). Using Rab7, Collage I, Collage III, $\alpha$ SMA, GAPDH (1:1000, Proteintech), MMP-14(1:1000, Bioworld, St. Louis Park, MN, USA) and RECK (1:1000, CST) antibodies as primary antibodies and adding second antibody. Protein supernatants were separated via 10\% SDS-PAGE (Invitrogen; Thermo Fisher Scientific, Inc.) and transferred to nitrocellulose membranes (placed in ice water with a constant current of $100 \mathrm{~mA}$ overnight). The antibody had been blocked with $5 \%$ skim milk at $4{ }^{\circ} \mathrm{Cover}$ night. An enhanced chemiluminescence(ECL) imaging method was used to detect the protein bands and the results were analyzed by Image $\mathrm{J}$ Software (Version 1.46, NIH, Bethesda, MD, USA). The experiment had been repeated for 3 times.

\section{Gelatin Zymography}

Collecting the kidney tissues and HMEC-1 of difference groups. The activity of MMP-2 in the condition media and the supernatants of the endothelial cells was 
examined by gelatin zymography (Applygen, China) ${ }^{[16]}$. The supernatants were collected after centrifuging and the protein content in the supernatants was detected by a BCA assay kit (Beyotime Biotechnology, China). The equal proteinamount of supernatants $(40 \mu \mathrm{g})$ were separated by $8 \%$ poly acryl amide gel containing $0.1 \%$ bovine gelatin (Sigma, USA). After electrophoresis, the gel had been transferred to $2.5 \%$ Triton $\mathrm{X}-100$ solution $(5 \mathrm{~h}$, twice) at room temperature and then had been incubated in Tris$\mathrm{HCl}$ buffer (pH 7.4) overnight. Next, the gel was treated with $0.1 \%$ Coomassie Brilliant Blue R-250, and white bands were visualized after the gel was destained in $30 \%$ methanol and $10 \%$ acetic acid and the results were analyzed by Image $J$ Software. The experiment had been repeated for 3 times.

\section{Sirius red stain}

After euthanasia, the kidneys were immediately removed, and a portion of each kidney was removed and frozen in liquid nitrogen. The remaining kidney tissue was fixed in $4 \%$ par formaldehyde, embedded in paraffin and sectioned. Sirius red staining was used to evaluate renal fibrosis in different groups.

\section{Immunohistochemistry stain}

Using anti-CD34 antibody to reveal peritubular capillary density in the interstitium. Incubating Specimens by antibody (1:400; Proteintech, Rosemont, IL, USA) at $4{ }^{\circ} \mathrm{C}$ overnight. Using anti- CD34 primary antibody by one-step polymer detection kit (Maixin, Fuzhou, China). After that, observing and evaluating results.

\section{Transmission electron microscope observition}

Taken the kidney tissues from difference groups, fixing by $2.5 \%$ glutaraldehyde (Sigma, USA) $\left(4^{\circ} \mathrm{C}, 24 \mathrm{~h}\right)$, and then post-fixed using $2 \%$ osmium tetroxide at room temperature. Specimens were cut into ultrathin sections $(50-70 \mathrm{~nm})$ and stained with uranyl acetate/lead citrate. Finally, ultra-structural analysis was performed under the JEM-1011EX instrument (JEOL, Japan).

\section{In situ gelatinase spectrum}

Frozen sections of fresh kidney tissue, with a thickness of $10 \mathrm{~nm}$, were adhered to the in-situ gelatinase membrane, placed in a wet box and incubated at $37{ }^{\circ} \mathrm{C}$ for $36 \mathrm{~h}$; the frozen sections were taken out and dried at room temperature for $30 \mathrm{~min}$, and then dyed in amino black 10B (1\% amino black, $70 \%$ methanol, $10 \%$ ethanol) for $15 \mathrm{~min}$, washed with distilled water, immersed in decolorizing solution $(70 \%$ methanol, $10 \%$ acetic acid) for $20 \mathrm{~min}$, and then dried. Finally, images were captured by laser scanning con focal microscopy (Olympus Corporation, Japan), evaluating MMP-2 activity of difference groups.

\section{Statistical analysis}

All experimental data were analyzed using SPSS 18.0 Software (SPSS, Inc., Chicago, IL, USA) and presented as the mean \pm standard deviation. Comparisons between groups were performed using one-way ANOVA with Bonferroni's post hoc correction (SPSS 18.0, IBM, Chicago, IL, USA) for multiple comparisons. $P<0.05$ was considered to indicate a statistically significant difference.

\section{Results}

Fibrosis level and CD34 protein expression in kidney tissues

By Sirius red staining, compared with NC group which was WT mice, the collage 
area was significantly increased in kidney fibrosis model mice $(\mathrm{P}<0.001$, Figure $1 \mathrm{~A})$; Compared with Model group, the collage area of Model+Rab7- which used Rab 7 knockdown mice was significantly depressed compared with that of Model group $(\mathrm{P}<$ 0.001 , Figure 1A). By IHC assay, compared with NC group, CD34 protein IOD was significantly down-regulation in Model group $(\mathrm{P}<0.001$, Figure 1B), Compared with Model group, CD34 protein IOD of Model+Rab7- which used Rab 7 knockdown mice was significantly up-regulation in kidney tissues $(\mathrm{P}<0.001$, Figure $1 \mathrm{~B})$.

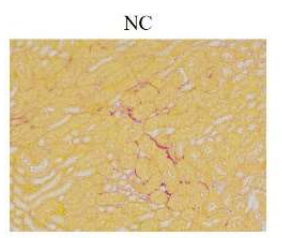

Model+Rab7-

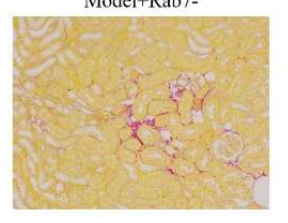

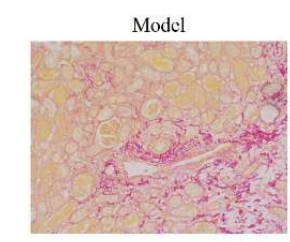

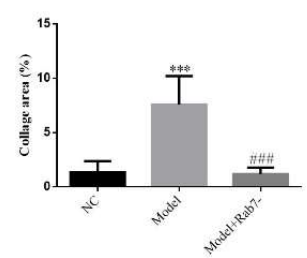

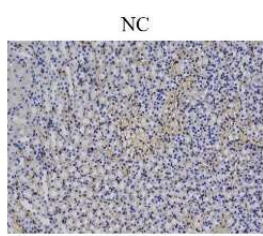

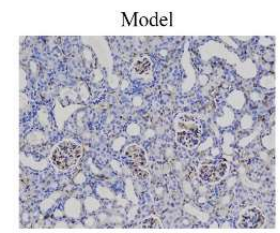

Model+Rab7-
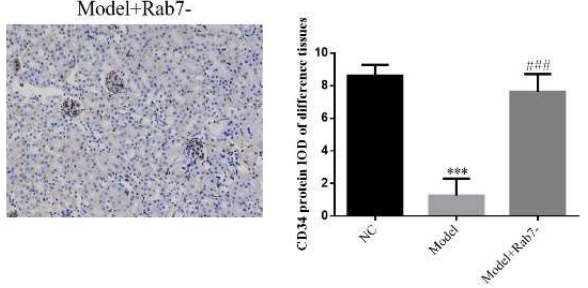

Figure 1. Collage area and CD34 protein expression in kidney tissues of difference groups NC: Normal mice; Model: kidney fibrosis model in normal mice; Model+Rab7-: kidney fibrosis model in Rab 7 knockdown mice

A. Collage area of difference groups by Sirius red stain $(200 \times)$

***: $\mathrm{P}<0.001$, compared with $\mathrm{NC}$ group; \#\#\#: $\mathrm{P}<0.001$, compared with Model group

B. CD34 protein expression IOD of difference tissues by IHC assay $(200 \times)$

***: $\mathrm{P}<0.001$, compared with NC group; \#\#\#: $\mathrm{P}<0.001$, compared with Model group

The ultrastructural changes of kidney tissues

By TEM observation, the ultrastructural was normal in kidney tissue of NC group, after kidney fibrosis molding, the ultrastructural was charge which the mitochondria and nuclei were damaged; however, the mitochondria and nuclei damaging were not found in another model group (Model+Rab 7-) which used Rab 7 knockdown mice's kidney tissues. The relative data was shown in Figure 2. 

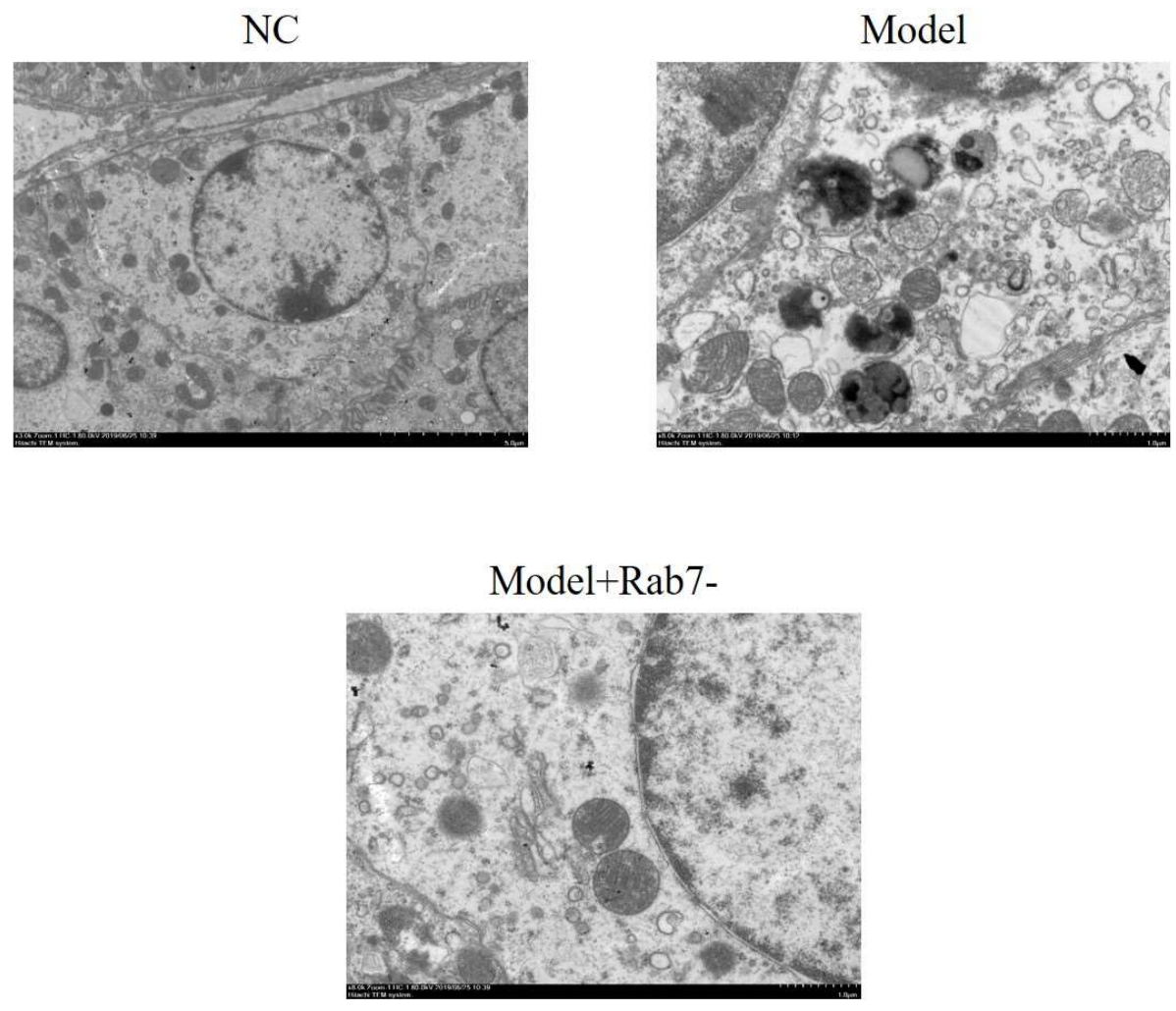

Figure 2. The ultrastructural changes of kidney tissues

NC: Normal mice; Model: kidney fibrosis model in normal mice; Model+Rab7-: kidney fibrosis model in Rab 7 knockdown mice

MMP-2 activities of difference groups

By In situ gelatinase spectrum, compared with NC group, MMP-2 activity of Model group was significantly depressed in tissues $(\mathrm{P}<0.001$, Figure $3 \mathrm{~A})$, however, with Rab 7 knockdown, compared with Model group, MMP-2 activity of Model+Rab7group was significantly increased in tissues $(\mathrm{P}<0.001$, Figure $3 \mathrm{~A})$. By Gelatin Zymography, compared with NC group, MMP-2 activity of Model group was significantly depressed in tissues $(\mathrm{P}<0.001$, Figure 3B), however, with Rab 7 knockdown, compared with Model group, MMP-2 activity of Model+Rab7- group was significantly increased in tissues $(\mathrm{P}<0.001$, Figure $3 \mathrm{~B})$. 


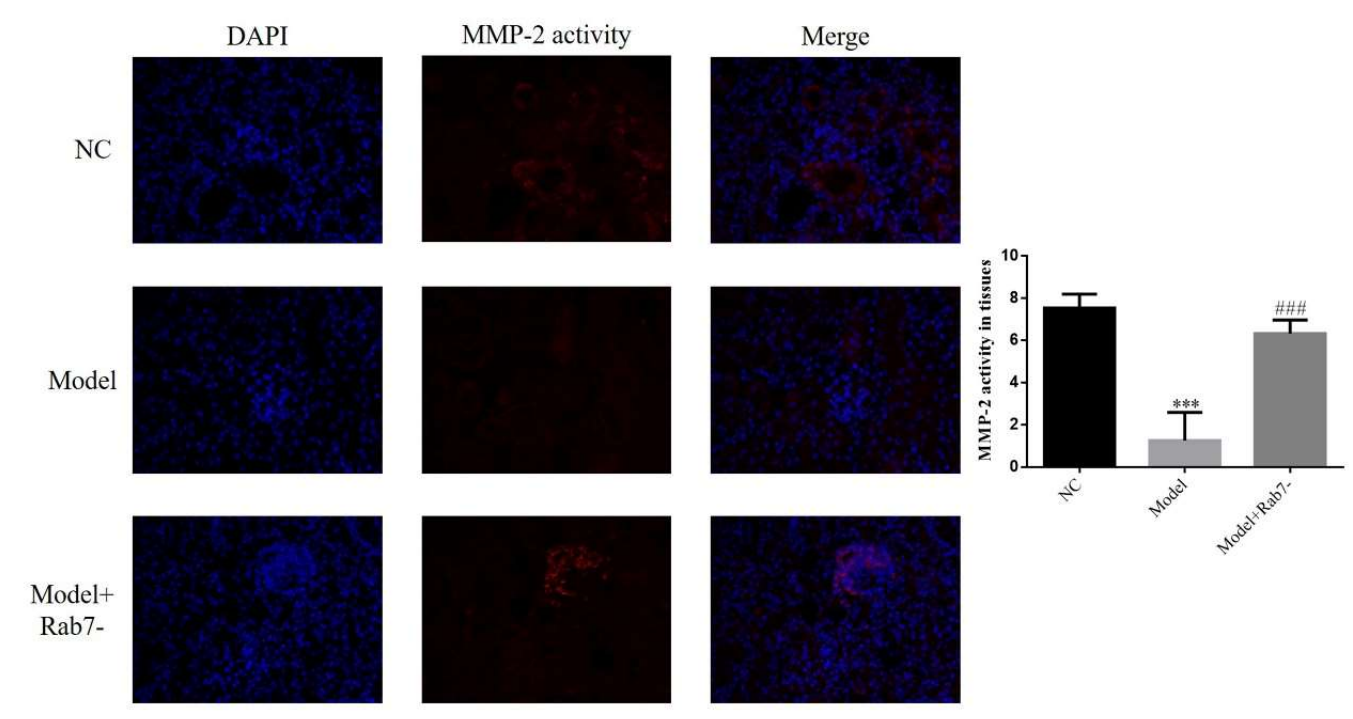

B
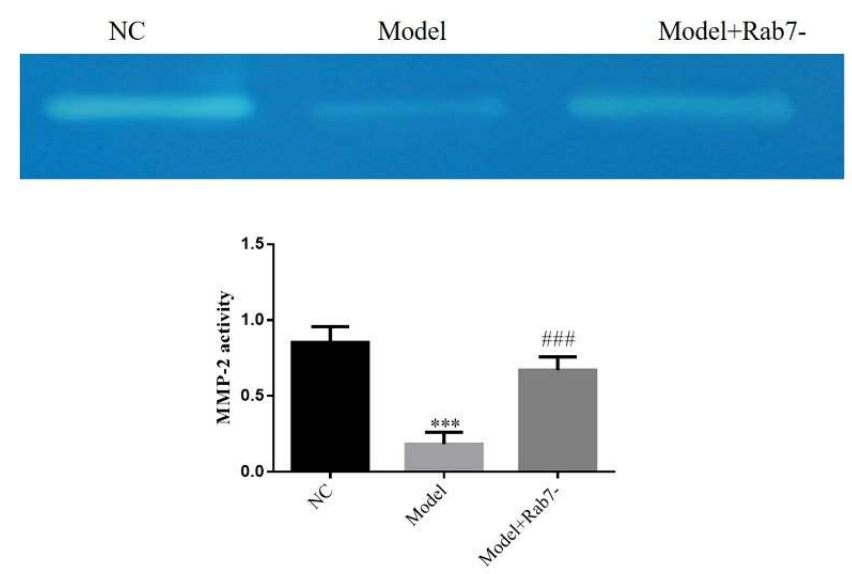

Figure 3. MMP-2 activities of difference groups

NC: Normal mice; Model: kidney fibrosis model in normal mice; Model+Rab7-: kidney fibrosis model in Rab 7 knockdown mice

A. MMP-2 activities of difference groups by In situ gelatinase spectrum $(200 \times)$

***: $\mathrm{P}<0.001$, compared with NC group; \#\#\#: $\mathrm{P}<0.001$, compared with Model group

B. MMP-2 activities of difference groups by Gelatin Zymography

***: $\mathrm{P}<0.001$, compared with NC group; \#\#\#: $\mathrm{P}<0.001$, compared with Model group

Relative protein expression in kidney tissues by WB assay

By WB assay, compared with NC group, the fibrosis marker proteins ( $\alpha$-SMA, Collage I and Collage III) and Rab 7 and RECK proteins expression were significantly up-regulation and MMP-14 protein expression was significantly down-regulation in Model group ( $\mathrm{P}<0.001$, respectively, Figure 4), however, using Rab 7 knockdown mice to model kidney fibrosis, the fibrosis marker proteins ( $\alpha$-SMA, Collage I and Collage III) and Rab 7 and RECK proteins expression were significantly depressed and MMP-14 protein expression was significantly increased in Model+Rab 7- group $(\mathrm{P}<$ 
0.001, respectively, Figure 4).

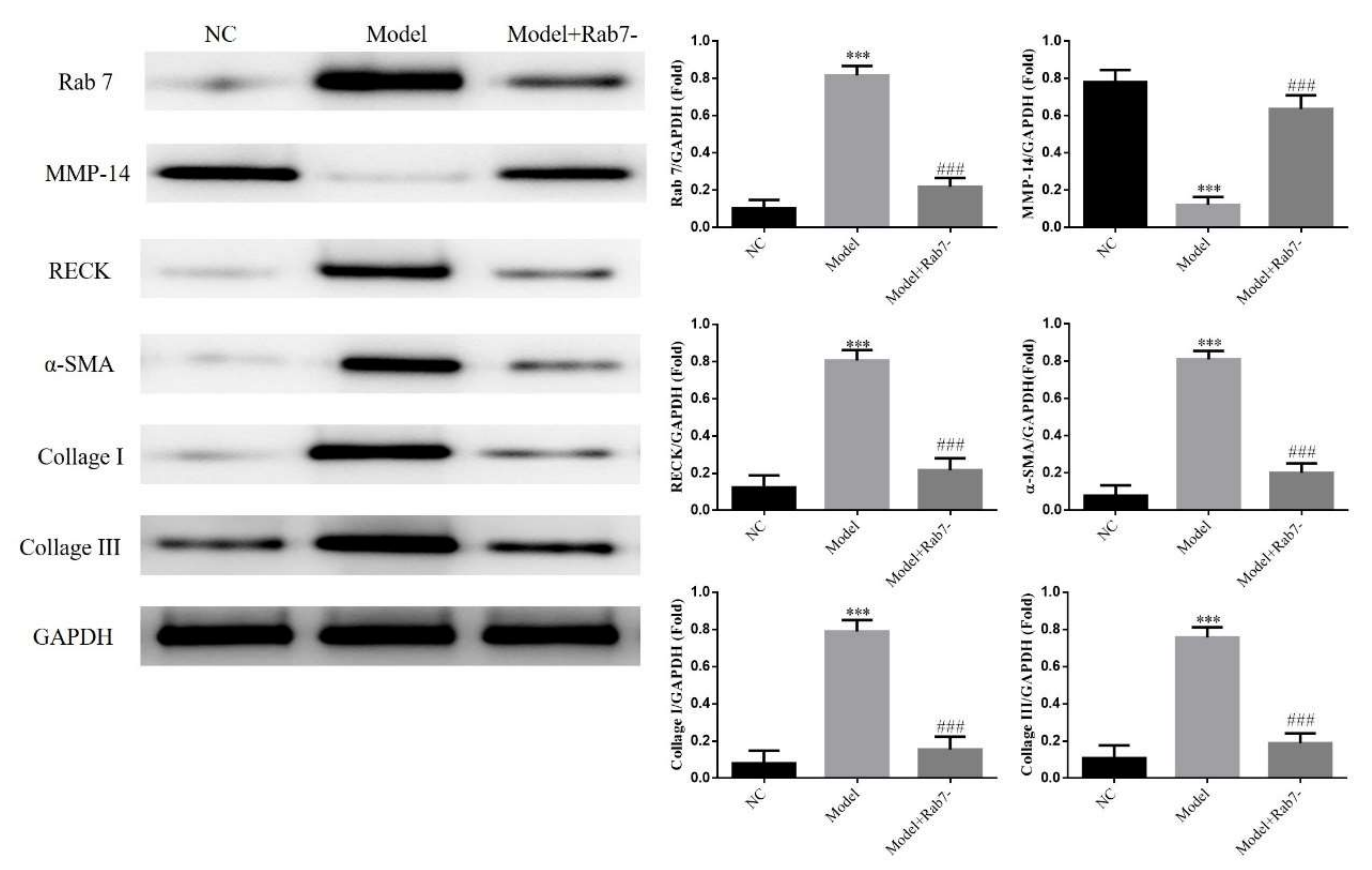

Figure 4. Relative proteins expression by WB assay

NC: Normal mice; Model: kidney fibrosis model in normal mice; Model+Rab7-: kidney fibrosis model in Rab 7 knockdown mice

***: $\mathrm{P}<0.001$, compared with NC group; \#\#\#: $\mathrm{P}<0.001$, compared with Model group

The cell proliferation and Ki67 positive cell number of difference cell groups

By CCK-8 assay, Compared with NC group, the cell proliferation rate of Model group was significantly depressed in $1 \mathrm{~h}, 2 \mathrm{~h}$ and $3.5 \mathrm{~h}(\mathrm{P}<0.05$, respectively, Figure 5A), with Rab 7 knockdown by si-Rab 7 supplement, the cell proliferation rate of Model+si-Rab 7 group was significantly recovery compared with Model group $(\mathrm{P}<$ 0.05 , respectively, Figure 5A) in $1 \mathrm{~h}, 2 \mathrm{~h}$ and $3.5 \mathrm{~h}$. Based on Model+si-Rab 7 group treatment, adding MMP-2 inhibitor, compared with Model+si-Rab 7 group, the cell proliferation rate of Model+si-Rab 7+MMP2 inhibitor was significantly downregulation $(\mathrm{P}<0.05$, respectively, Figure $5 \mathrm{~A})$ in $1 \mathrm{~h}, 2 \mathrm{~h}$ and $3.5 \mathrm{~h}$. In order to observant cell activity, we measured Ki67 protein expression by immunofluorescence, Compared with NC group, Ki67 positive cell number of Model and Model+si-NC groups were significantly down-regulation ( $\mathrm{P}<0.001$, respectively, Figure $5 \mathrm{~B}$ ), however, with siRab 7 supplement which knockdown Rab 7, compared with Model group, the Ki67 positive cell number of Model+si-Rab 7 group was significantly up-regulation $(\mathrm{P}<$ 0.001, respectively, Figure 5B), meanwhile, with MMP2 inhibitor supplement, compared with Model+si-Rab 7 group, Ki67 positive cell number of Model+si-Rab $7+\mathrm{MMP} 2$ inhibitor group was significantly depressed $(\mathrm{P}<0.001$, Figure $5 \mathrm{~B})$. 


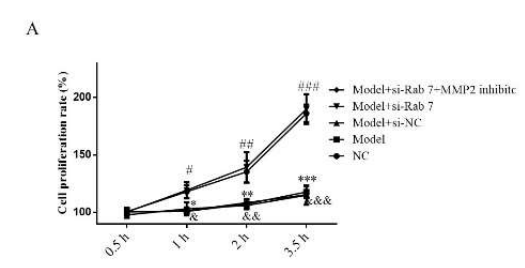

B
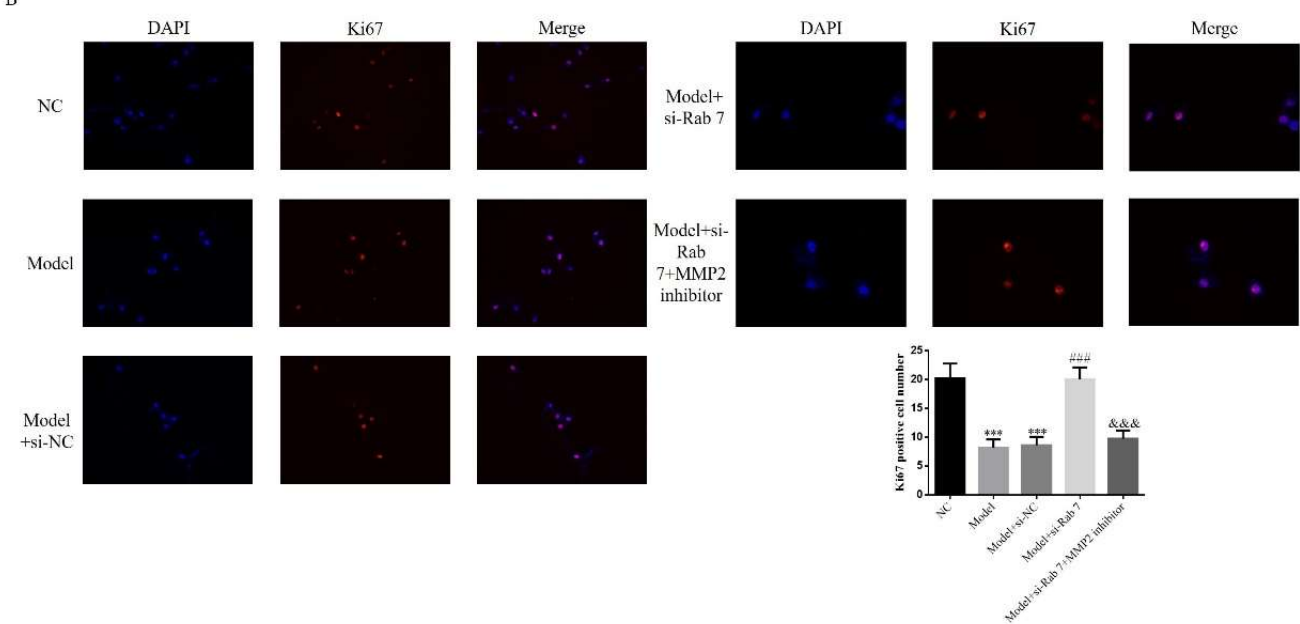

Figure 5. Cell proliferation and Ki67 positive cell number

A. Cell proliferation in difference times points

*: $\mathrm{P}<0.05, * *: \mathrm{P}<0.01, * * *: \mathrm{P}<0.001$, compared with $\mathrm{NC}$ group; \#: $\mathrm{P}<0.05$, \#\#: $\mathrm{P}<$ 0.01, \#\#\#: $\mathrm{P}<0.001$, compared with Model group; \&: $\mathrm{P}<0.05$, \&\&: $\mathrm{P}<0.001$, \&\&\&: $\mathrm{P}$ $<0.001$, compared with Model + si-Rab 7

B. Ki37 positive cell number of difference groups

***: $\mathrm{P}<0.001$, compared with $\mathrm{NC}$ group; \#\#\#: $\mathrm{P}<0.001$, compared with Model group; $\& \& \&: \mathrm{P}<0.001$, compared with Model + si-Rab 7 group

Rab 7 affect HMEC-1 cell's migration ability

By transwell assay, compared with $\mathrm{NC}$ group, migration cell number of Model and Model + si-NC group were significantly suppressed $(\mathrm{P}<0.001$, respectively, Figure 6$)$; with Rab 7 knockdown, compared with Model group, migration HMEC-1 cell number of Model+si-Rab 7 group was significantly up-regulation $(\mathrm{P}<0.001$, Figure 6$)$; with MMP2 inhibitor supplement, compared with Model+si-Rab 7 group, migration HMEC1 cell number of Model + si-Rab 7+MMP2 inhibitor group was significantly downregulation $(\mathrm{P}<0.001$, Figure 6$)$. 

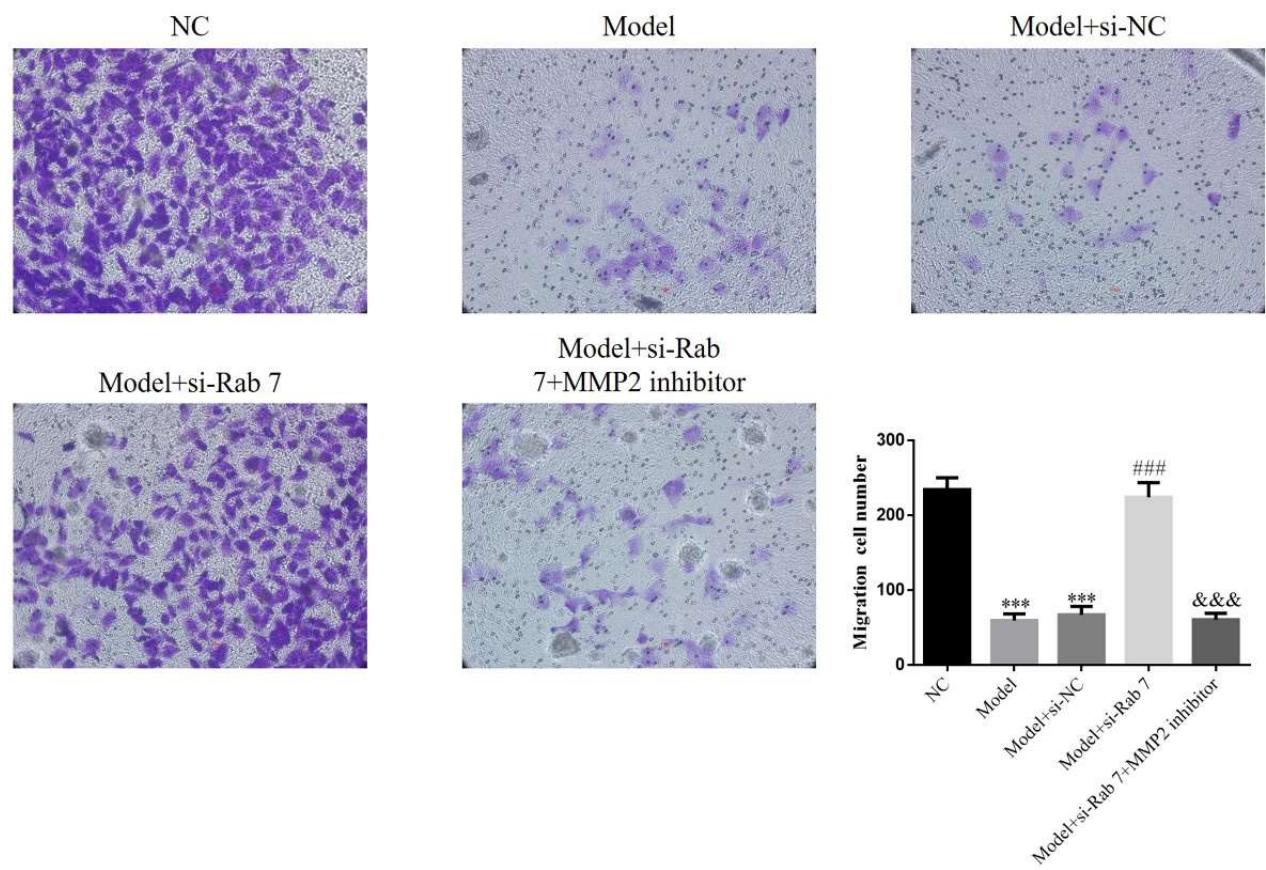

Figure 6. Migration cell number of difference groups ***: $\mathrm{P}<0.001$, compared with $\mathrm{NC}$ group; \#\#\#: $\mathrm{P}<0.001$, compared with Model group; $\& \& \&: \mathrm{P}<0.001$, compared with Model + si-Rab 7 group

Total length of difference groups by vitro angiogenesis

By vitro angiogenesis, compared with NC group, total length of Model and Model + si-NC groups were significantly down-regulation $(\mathrm{P}<0.001$, respectively, Figure 7), with Rab 7 knockdown, compared with Model group, total length of Model + si-Rab 7 group was significantly up-regulation $(\mathrm{P}<0.001$, Figure 7$)$, however, with MMP2 inhibitor supplement, compared with Model+si-Rab 7 group, total length of Model+si-Rab 7+MMP2 inhibitor group was significantly down-regulation $(\mathrm{P}<$ 0.001 , Figure 7). 

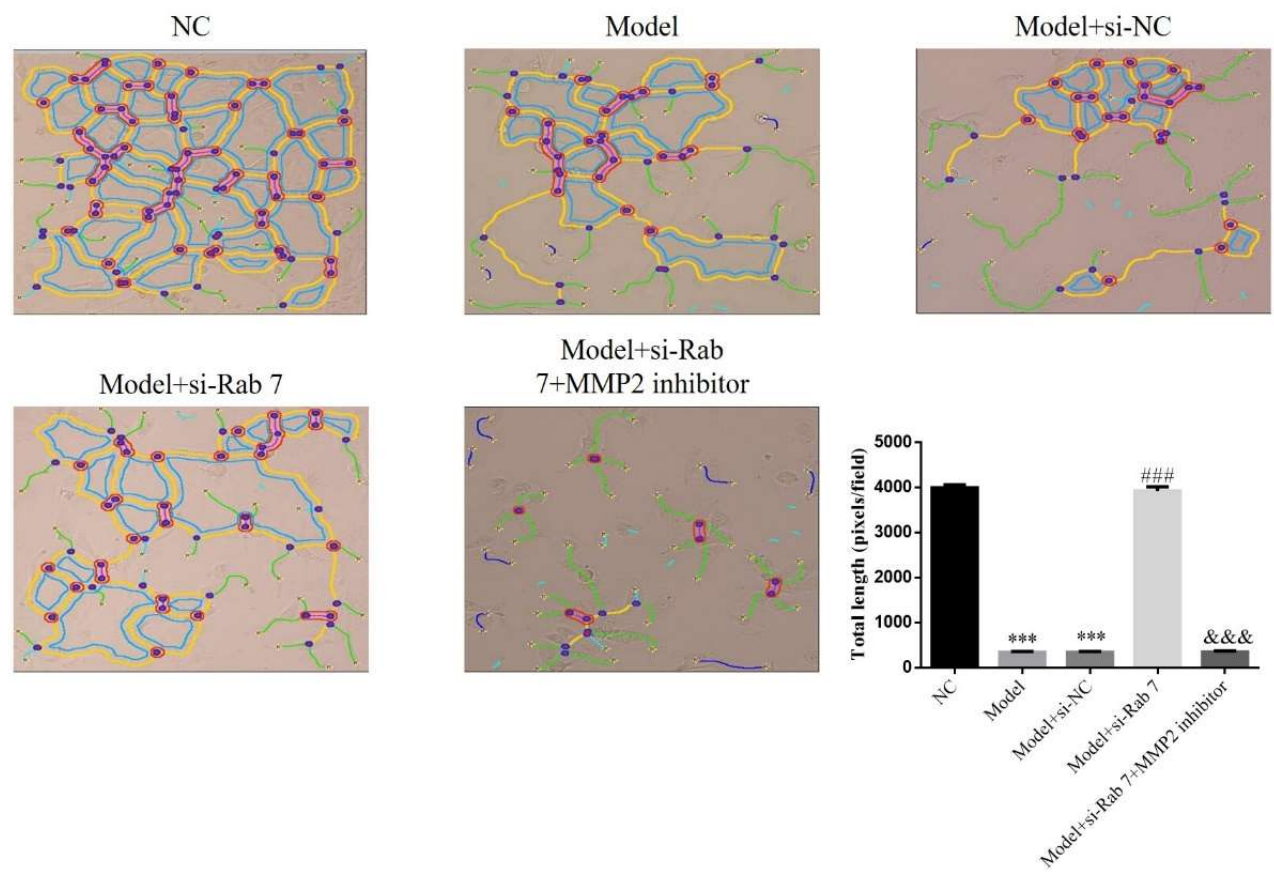

Figure 7. Total length of difference group ***: $\mathrm{P}<0.001$, compared with NC group; \#\#\#: $\mathrm{P}<0.001$, compared with Model group; $\& \& \&: \mathrm{P}<0.001$, compared with Model+si-Rab 7 group

Relative proteins expression by WB assay

By WB assay, compared with NC group, fibrosis marker proteins ( $\alpha$-SMA, Collage I and Collage III) and RECK and Rab 7 proteins expressions were significantly increased, and MMP-14 protein expression was significantly depressed $(\mathrm{P}<0.001$, respectively, Figure 8) in Model and Model+si-NC group; with Rab 7 knockdown, compared with Model group, fibrosis marker proteins ( $\alpha$-SMA, Collage I and Collage III) and RECK and Rab 7 proteins expressions were significantly depressed, and MMP14 protein expression was significantly increased $(\mathrm{P}<0.001$, respectively, Figure 8$)$ in Model+si-Rab 7 group; meanwhile, with MMP2 inhibitor supplement, compared with Model + si-Rab 7 group, fibrosis marker proteins ( $\alpha$-SMA, Collage I and Collage III) and RECK and Rab 7 proteins expressions were significantly increased, and MMP-14 protein expression was significantly depressed $(\mathrm{P}<0.001$, respectively, Figure 8$)$ in Model+si-Rab 7+MMP2 inhibitor group. 


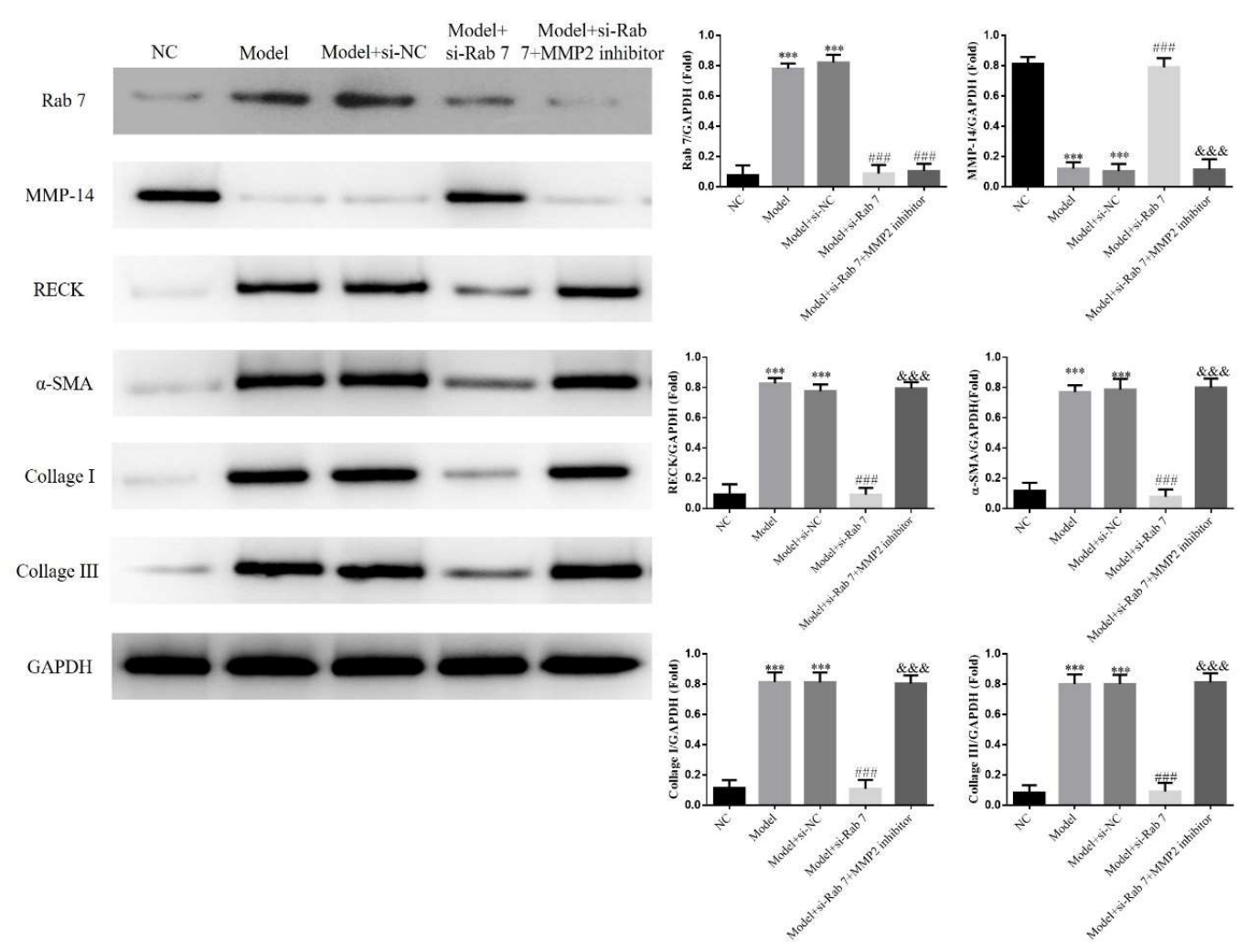

Figure 8 . Relative protein expression by WB assay

***: $\mathrm{P}<0.001$, compared with NC group; \#\#\#: $\mathrm{P}<0.001$, compared with Model group; $\& \& \&: \mathrm{P}<0.001$, compared with Model + si-Rab 7 group

MMP2 activity by Gelatin Zymography in vitro

Compared with NC group, MMP2 activity of Model and Model+si-NC groups were significantly down-regulation $(\mathrm{P}<0.001$, respectively, Figure 9), with Rab 7 knock down, compared with Model group, MMP2 activity of Model+si-Rab 7 group was significantly up-regulation ( $\mathrm{P}<0.001$, Figure 9); with MMP2 inhibitor, compared with Model+si-Rab 7 group, MMP2 activity of Model+si-Rab 7+MMP2 inhibitor group was significantly down-regulation $(\mathrm{P}<0.001$, Figure 9$)$. 


$$
\text { NC Model Model+si-NC } \begin{array}{lc}
\text { Model+ } & \text { Model+si-Rab } \\
\text { si-Rab 7 }
\end{array} \quad \begin{aligned}
& \text { 7+MMP-2 inhibitor }
\end{aligned}
$$
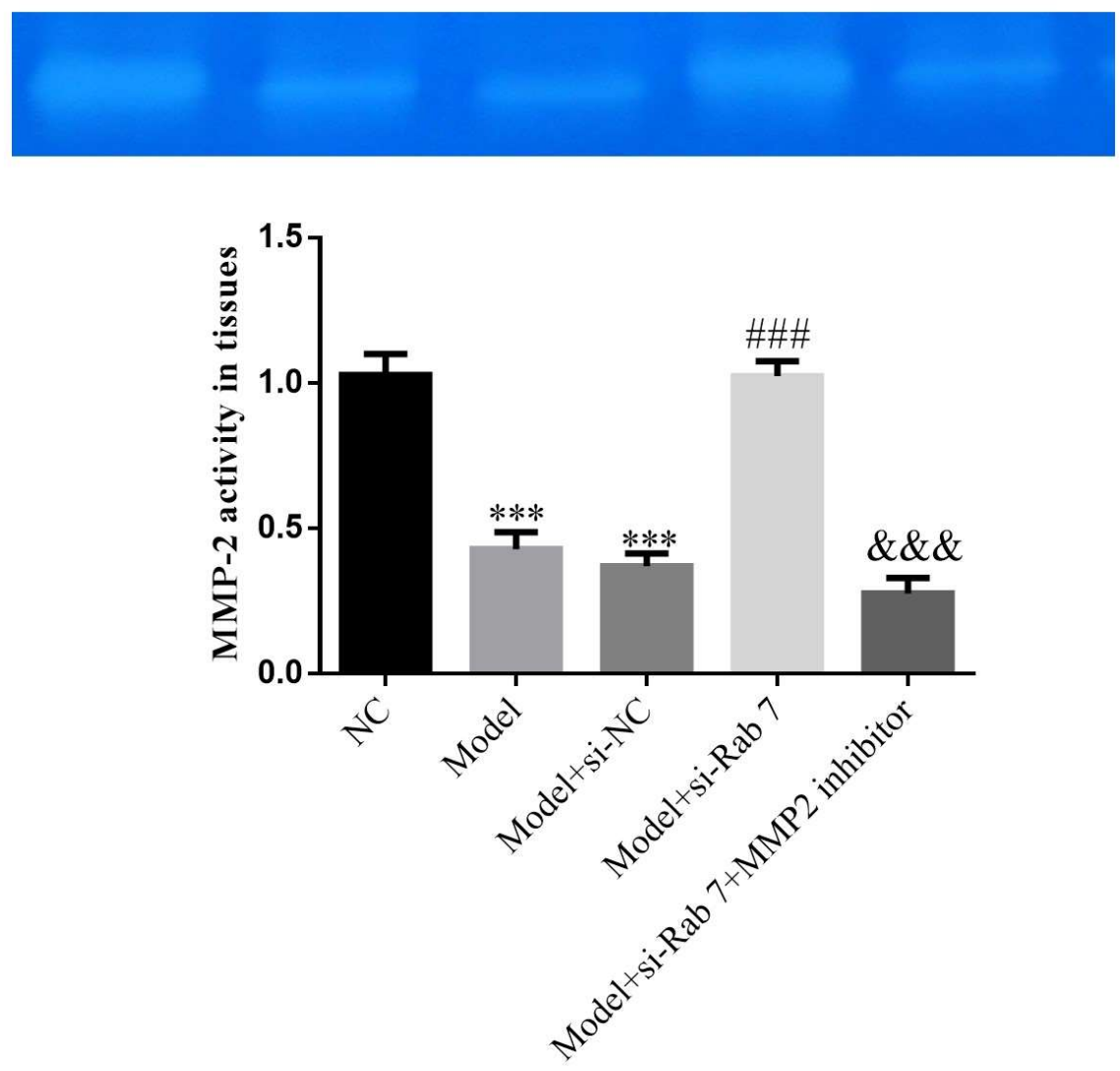

Figure 9. MMP-2 activities of difference groups by Gelatin Zymography

***: $\mathrm{P}<0.001$, compared with NC group; \#\#\#: $\mathrm{P}<0.001$, compared with Model group; $\& \& \&: \mathrm{P}<0.001$, compared with Model + si-Rab 7 group

\section{Discussion}

Peritubular capillary rarefaction is an important pathological feature of CKD and the main cause of renal hypoxia and hypoxia promotes the development of CKD ${ }^{[17]}$. Therefore, promoting the formation of peritubular capillaries and improving tissue oxygen supply are basic strategies to delay or even reverse the development of $\mathrm{CKD}^{[18]}$.Hypoxia is a prominent pathophysiological feature of renal fibrotic tissue. A series of studies of our group have confirmed that supernatant of hypoxic cultured renal tubular epithelial cells and renal fibrotic tissues of animal model show decreased enzyme activity of MMP-2 ${ }^{[2,8]}$, but the mechanism of decreased enzyme activity under hypoxia condition needs to be clarified.

MMP-2 is mainly released from cells in form of proenzyme and the activation on the surface of cells $[19,23]$. Since the activation of MMP-2 is mainly on the surface of plasma membrane, we concluded that the changes of cell membrane structure could affect the activity of this enzyme. Autophagy and endocytosis are not only the main factors of cell membrane remodeling, but also play a decisive role in the regulation of transmembrane signal transduction and a variety of metabolic or functional activities[24-26]. Previous study of our group confirmed that autophagy and endocytosis of hypoxic renal tubular epithelial cells do affect the activity of MMP-2 
[15].

Previous studies have confirmed that the co-occurrence of endocytosis and autophagy under hypoxia conditions arise from the sharing of some functional links [15].As a shared molecule of autophagy and endocytosis in renal tubular epithelial cells, Rab7 can control the transport of late endocytosis and autophagy to lysosomes[27,28].Rab7 plays an important role in pinocytosis, phagocytosis, exocytosis and autophagy (including mitochondrial autophagy and lipid droplet autophagy). Meanwhile, Rab7 is the basis of lysosomal biosynthesis, localization and function, and also participates in many physiological activities such as apoptosis, neurotrophic factor transport and signal transduction, axon growth and tumor inhibition [29]. Rab7 is a hinge molecule linking various functions inside and outside of the cell [30].

In this study, the effect of Rab7 expression on angiogenesis in hypoxic renal tubular epithelial cells was studied in vivo and vitro, and we explored whether Rab7 regulated peritubular capillary by regulating the activity of MMP-2in hypoxic renal tubular epithelial cells. In vivo study, we found that fibrosis level, pathological injury and mitochondrial damage were aggravated in kidney tissues of kidney fibrosis model mice. However, fibrosis level, pathological injury and mitochondrial damage were significantly improved in Rab 7 knockdown mice with kidney fibrosis modeling. In vitro study, the results found that endothelial cells (HMEC-1) co-confirmed with HK-2 cell which knock down Rab 7 by si-Rab 7 were depressed cell proliferation, migration and tubular formation.

In order to confirm whether Rab7 has an effect on angiogenesis through regulating the activity of MMP-2, we used ARP100 to inhibitedthe activity of MMP-2and repeated the above experiment. The results of gelatin zymography assay showed that MMP2 activity was significantly decreased in both normoxia and a hypoxia group, indicating that ARP100 interference was effective. At the same time, it was found that the conditioned media with different expression levels of Rab7 lost its influence on proliferation, migration and tubular formation of endothelial cells in hypoxic and normoxic condition. This study demonstrated that the high expression of Rab7 in hypoxic renal tubular epithelial cells could play an anti-angiogenic role by inhibiting the activity of MMP-2.

In this study, we also found an interesting phenomenon, that is, the cultured supernatant of renal tubular epithelial cells with low expression of Rab7 promotes the proliferation of endothelial cells, which has not been reported, so it is necessary to explore the internal mechanism in subsequent experiments. In recent years, some scholars have carried out some research on renal agiogenesis. Zhu and other found that the mesenchymal stromal cell-derived extracellular vesicles alleviated renal ischemic reperfusion injury and enhance angiogenesis in rats [33]. Chade used a bioengineered polymer-stabilized vascular endothelial growth factor to promote renal angiogenesis [34]. And it was reported that protein kinase LKB1 promoted Rab7-mediated NRP-1 degradation and inhibits tumor angiogenesis [35]. In previous research, we found that endothelial cells co-cultured with renal carcinoma cells significantly reduced RECK expression under chemical hypoxia[36].As can be seen from these reports, the mechanism of angiogenesis is complex in kidney diseases, perhaps it shares different pathways between tumors and inflammations or between early stage and later stage of the diseases.

In conclusion,we confirmed that the over-expression of Rab7 in hypoxic renal tubular epithelial cells interfered with the generation of peritubular capillaries by regulating the activity of MMP-2in vivo and vitro experiments. In our opinion, targeted 
down-regulation of Rab7 expression may be a candidate for delay the development of chronic kidney disease.

\section{References}

1. Shen H, van der Kleij RMJJ, van der Boog PJM, Chang X, Chavannes NH. Electronic Health Self-Management Interventions for Patients With Chronic Kidney Disease: Systematic Review of Quantitative and Qualitative Evidence. J Med Internet Res. 2019; 21(11):e12384.

2. Cheng Z, Liu L, Wang Z, Cai Y, Xu Q and Chen P. Hypoxia activates Src and promotes endocytosis which decreases MMP-2 activity and aggravates renal interstitial fibrosis. Int J Mol Sci. 2018; 19(2):581.

3. Liu BC, Tang TT, Lv LL and Lan HY. Renal tubule injury: a driving force toward chronic kidney disease. Kidney Intel. 2018; 93(3):568-579

4. Tanaka S, Tanaka T, Nangaku M. Hypoxia and dysregulated angiogenesis in kidney disease. Kidney Dis. 2015; 1:80-89.

5. Masum M A, Ichii O, Elewa YHA, Nakamura T, Kon Y. Local CD34-positive capillaries decrease in mouse models of kidney disease associating with the severity of glomerular and tubulointerstitial lesions. BMC Nephrol. 2017; 18(1):280

6. Kida Y, Tchao BN, Yamaguchi I. Peritubular capillary rarefaction: a new therapeutic target in chronic kidney disease. PediatrNephrol. 2014; 29(3):333-342

7. Carmeliet P \& Jain R K. Molecular mechanisms and clinical applications of angiogenesis. Nature.2011; 473: 298-307.

8. Ding F, Li Y, Liu J, Liu L, Yu W, Wang Z, Ni H, Liu B, Chen P. Overendocytosis of gold nanoparticles increases autophagy and apoptosis in hypoxic human renal proximal tubular cells. Int J Nanomed. 2014;9: 4317-4330.

9. Henriet P, Emonard H. Matrix metalloproteinase-2: Not (just) a "hero" of the past.Biochimie. 2019;166:223-232.

10. Cheng Z, Limbu MH, Wang Z, Liu J, Liu L, Zhang X, Chen P and Liu B. MMP-2 and 9 in Chronic Kidney Disease. Int J Mol Sci. 2017;18(4): 776.

11. Pinilla-Macua I, Grassart A, Duvvuri U, Watkins SC, Sorkin A. EGF receptor signaling, phosphorylation, ubiquitylation and endocytosis in tumors in vivo. ELife. 2017;6 :e31993.

12. Gutierrez MG, Munafó DB, Berón W, Colombo MI. Rab7 is required for the normal progression of the autophagic pathway in mammalian cells. J Cell Sci, 2004,117(Pt 13):2687-2697.

13. Hyttinen JMT, Niittykoski M, Salminen A, Kaarniranta K. Maturation of autophagosomes and endosomes: A key role for Rab7. BiochimBiophysActa. 2013;1833(3):503-510

14. Yu W, Wang Z, Liu L, Liu J, Ding F, Zhang X, Cheng Z, Li Y, Chen P, Dou J. Endocytosis mediated by Caveolin-1 inhibits activity of matrix metalloproteinase- 2 in human renal proximal tubular cells under hypoxia. Int J ClinExpPathol. 2016;9(2): 1276-1284

15. Yu W, Wang Z, Li Y, Liu L, Liu J, Ding F, Zhang X, Cheng Z, Chen P. Effects of autophagy and endocytosis on the activity of matrix metalloproteinase 2 in human renal proximal tubular cells under hypoxia. Mol Med Rep. 2017;15(5):3225-3230.

16. Toth M, Sohail A, Fridman R. Assessment of gelatinases (MMP-2 and MMP-9) by gelatin zymography. Methods Mol Biol. 2012;878:121-35.

17. Mayer G. Capillary rarefaction, hypoxia, VEGF and angiogenesis in chronic renal disease.Nephrol Dial Transplant 2011; 26: 1132-1137. 
18.Ballermann B J, Obeidat M. Tipping the balance from angiogenesis to fibrosis in CKD. Kidney Int Suppl. 2014;4(1):45-52.

19. Mahalanobish S, Saha S, Dutta S, Sil PC. Matrix metalloproteinase: An upcoming therapeutic approach for idiopathic pulmonary fibrosis. Pharmacol Res. 2020;152: 104591.

20. Dimas GG, Didangelos TP, Grekas DM. Matrix Gelatinases in Atherosclerosis and Diabetic Nephropathy: Progress and Challenges. CurrVascPharmacol2017;15(6):557565.

21. Narula S, Tandon C, Tandon S. Role of Matrix Metalloproteinases in Degenerative Kidney Disorders. Curr Med Chem. 2018;25(15):1805-1816.

22. Parrish AR. Matrix Metalloproteinases in Kidney Disease: Role in Pathogenesis and Potential as a Therapeutic Target. ProgMolBiolTranslSci 2017;148:31-65.

23. Provenzano M, Andreucci M, Garofalo C, Faga T, Michael A,et al. The Association of Matrix MetalloproteinasesWith Chronic Kidney Disease and Peripheral Vascular Disease: A Light at the End of the Tunnel? Biomolecules 2020; 10(1):154.

24. Mahapatra KK, Panigrahi DP, Praharaj PP, Bhol CS, Patra S, Mishra SR, Behera BP, Bhutia SK. Molecular interplay of autophagy and endocytosis in human health and diseases.Biol Rev CambPhilos Soc. 2019;94(4):1576-1590.

25. Yu L, Chen Y, Tooze SA. Autophagy pathway: Cellular and molecular mechanisms. Autophagy. 2018;14(2):207-215.

26. Zhang HL, Zhu YM, Zhou XY. Coordination of Autophagy and Other Cellular Activities.AdvExp Med Biol. 2019; 1206:697-727.

27. Wong YC, Ysselstein D, Krainc D. Mitochondria-lysosome contacts regulate mitochondrial fission via RAB7 GTP hydrolysis. Nature. 2018;554(7692):382-386.

28. Stroupe C. This Is the End: Regulation of Rab7 Nucleotide Binding in Endolysosomal Trafficking and Autophagy. Front Cell Dev Biol. 2018; 6:129.

29. Guerra F, Bucci C. Multiple Roles of the Small GTPase Rab7. Cells. 2016;5(3):34. 30. Roque NR, Lage SL, Navarro R, Fazolini N, Maya-Monteiro CM, Rietdorf J, Melo RCN, D'Avila H, Bozza PT. Rab7 controls lipid droplet-phagosome association during mycobacterial infection. BiochimBiophysActaMol Cell Biol Lipids.2020; 1865(8): 158703.

31. Jopling HM., Odell AF, Hooper N M, Zachary IC, Walker JH, Ponnambalam S. RabGTPase regulation of VEGFR2 trafficking and signaling in endothelial cells. ArteriosclerThrombVasc Biol. 2009;29:1119-1124.

32. Kofler N, Corti F, Rivera-Molina F, Deng Y, Toomre D and Simons M. The Rabeffector protein RABEP2 regulates endosomal trafficking to mediate vascular endothelial growth factor receptor-2 (VEGFR2)-dependent signaling. J Biol Chem. 2018;293(13):4805-4817

33. Zou X, Gu D, Xing X, Cheng Z, Gong D, Zhang G, Zhu Y. Human mesenchymal stromal cell-derived extracellular vesicles alleviate renal ischemic reperfusion injury and enhance angiogenesis in rats. Am J Transl Res 2016;8(10):4289-4299

34. Chade AR., Tullos NA., Harvey TW, Mnahdi F, and Bidwell III GL. Renal therapeutic angiogenesis using a bioengineered polymer-stabilized vascular endothelial growth factor construct. J Am SocNephrol 2016; 27: 1741-1752.

35. Okon IS, Coughlan KA, Zhang C, Moriasi C, Ding Y, Song P, Zhang W, Li G and Zou M-H. Protein kinase LKB1 promotes RAB7-mediated neuropilin-1 degradation to inhibit angiogenesis. J Clin Invest. 2014;124(10):4590-4602.

36. Zhang X, Liu L, Liu J, Cheng Z, Wang Z, Shi C, Ding F, Chen S, Chen P. Endothelial cells co-cultured with renal carcinoma cells significantly reduce RECK expression under chemical hypoxia. Cell BiolInt 2017; 9999:1-6. 